This item was submitted to Loughborough's Research Repository by the author.

Items in Figshare are protected by copyright, with all rights reserved, unless otherwise indicated.

\title{
Far-field noise prediction of round and serrated jets with increasingly refined grids
}

PLEASE CITE THE PUBLISHED VERSION

http://dx.doi.org/10.2514/6.2016-3047

PUBLISHER

(c) AIAA

VERSION

AM (Accepted Manuscript)

\section{PUBLISHER STATEMENT}

This work is made available according to the conditions of the Creative Commons Attribution-NonCommercialNoDerivatives 4.0 International (CC BY-NC-ND 4.0) licence. Full details of this licence are available at: https://creativecommons.org/licenses/by-nc-nd/4.0/

\section{LICENCE}

CC BY-NC-ND 4.0

\section{REPOSITORY RECORD}

Angelino, Matteo, Hao Xia, Miguel Moratilla-Vega, and Gary J. Page. 2016. "Far-field Noise Prediction of Round and Serrated Jets with Increasingly Refined Grids". figshare. https://hdl.handle.net/2134/21418. 


\title{
Far-field Noise Prediction of Round and Serrated Jets with Increasingly Refined Grids
}

\author{
M. Angelino*, H. Xia ${ }^{\dagger}$, M. A. Moratilla-Vega ${ }^{\ddagger}$ and G. J. Page ${ }^{\S}$ \\ Department of Aeronautical and Automotive Engineering, Loughborough University \\ Loughborough, Leicestershire, LE11 3TU, United Kingdom
}

\begin{abstract}
Turbulent jet large-eddy simulations (LES) are performed at Mach 0.9 and Reynolds number of $10^{6}$. For subgrid scale stress modeling the $\sigma$-model is used. Solutions are obtained for a baseline axisymmetric (round) nozzle and a serrated (or chevron) nozzle with high bending and penetration, on grids ranging from 5 to 80 million grid points in order to assess the correlation between coarser and finer grid solutions. Computed mean and second-order fluctuating quantities of the turbulent near field compare favorably with measurements. The radiated far-field sound is predicted using the Ffowcs Williams and Hawkings (FW-H) surface integral method. Remarkable agreement of the predicted farfield sound directivity and spectra with measurements is obtained. A preliminary discussion is presented on the correlation and possible combination of multiple spectra from different grids.
\end{abstract}

\section{Nomenclature}

a speed of sound

A Jacobian, $\mathbf{A}=\partial \mathbf{F} / \partial \mathbf{Q}$

$D$ diameter

F flux vectors

$n_{j} \quad$ normal vector's $j$-th component

$\nu \quad$ kinematic viscosity

$p^{\prime} \quad$ pressure fluctuation, acoustic pressure

Q vector of primitive variables

St Strouhal number

$t \quad$ physical time, $t^{*}=D_{j} / U_{j}$

$T$ temperature

$\mathrm{x} \quad$ Cartesian coordinates

$\Theta \quad$ FW-H observer angular position

Subscripts

$\infty$ ambient condition

$j \quad$ nozzle exit condition

$n$ outward normal direction

$S G S$ subgrid-scale

*Research Associate, Rolls-Royce University Technology Centre

${ }^{\dagger}$ Lecturer, Rolls-Royce University Technology Centre

${ }^{\ddagger} \mathrm{PhD}$ Student, Rolls-Royce University Technology Centre

§Professor, Rolls-Royce University Technology Centre 


\section{Introduction}

Numerical prediction of jet noise has received significant attention in the past decade as advances in high performance computing technology have given computational aeroacoustics more power ever than before. The desire to more reliably predict the noise reduction available to individual jet engine designs has led to the increasingly popular use of Large-Eddy Simulation. For example, Xia et al. ${ }^{1,2}$ recently made attempts to predict far-field noise radiated from chevron nozzles, Uzun and Hussuni ${ }^{3}$ showed high frequency spectra of a chevron jet, while Shur et al. ${ }^{4,5}$ and Mendez et al. ${ }^{6}$ explored a generic approach for emulating complex nozzle jets.

The key issue LES predictions are facing is the spectral range requirement of Strouhal number (the nondimensional frequency, $S t=f D_{j} / U_{j}$ ) from 0.05 to 8 for a medium size jet engine. LES have a limited range of the achievable frequencies due to limitations with current computational power on grid resolution and time sampling. As a result, the maximum reliable prediction of the Strouhal number using LES is considerably lower than $8 .^{7}$ With noise suppression designs such as chevrons ${ }^{8}$ and microjets, ${ }^{9}$ high frequency spectra are even more difficult to capture.

Simply increasing the LES spatial and temporal resolution does not seem to solve the problem. A wellresolved fine-grid solution, ${ }^{10}$ which may capture the high-frequency spectrum, is often too costly to run for a sufficiently long period of time to be statistically converged to capture the low frequencies (e.g. $S t \sim 0.05)$. In other words, the fine solution typically produces much better high-frequency predictions but performs poorly towards low frequencies, and the coarser solution spectrum decays far too quickly towards the high frequencies but does provide good low-frequency predictions simply because it is allowed to run for much longer physical time. The typical trend is suggested by many jet noise LES studies summarized in Bodony and Lele. ${ }^{7}$ For instance, high resolution grids of $100 \sim 370$ million points were used in Uzun, ${ }^{3}$ where the predicted noise spectra show good agreement with experiments towards the high frequency for almost up to $S t=10$ but compare poorly with experiments for $S t<1$ even with a sampling period of 150 non-dimensional time units. Similarly, using a coarser grid $\left(\sim 20\right.$ million points), Xia and Tucker ${ }^{1}$ were able to capture the low-frequency spectra, but their high frequency spectra showed a fast decay after $S t=3$. Evidently, this reflects the classic challenge of turbulent flow numerical simulation known as scale disparity. To be able to resolve the small scales (corresponding to high-frequency noise) and at the same time to be able to run sufficient time sampling to capture large scale motions (low frequency noise) is clearly preventing LES based methods from being further applied to far more complex and realistic industrial jet noise problems.

This paper provides a series of numerical observations on the spectra from multiple LES solutions with sequentially refined grids. The aim is to lay the foundations for a strategy to potentially limit the computational costs by combining low-frequency results from coarser grids with high-frequency results from finer grids.

\section{Numerical methods}

\section{II.A. Governing equations}

The Favre-average/filtered compressible Navier-Stokes equations for ideal gas are solved. The conservative form of the continuity, momentum and energy equations can be expressed as

$$
\frac{\partial \overline{\mathbf{Q}}}{\partial t}+\frac{\partial \overline{\mathbf{F}}_{i}^{i n v}}{\partial x_{i}}-\frac{\partial \overline{\mathbf{F}}_{i}^{v i s}}{\partial x_{i}}=0
$$

The conservative variables are defined as $\overline{\mathbf{Q}}=\left[\bar{\rho}, \bar{\rho} \tilde{u}_{i}, \tilde{E}\right]^{T}$. The inviscid and viscous fluxes are respec-

tively given by $\overline{\mathbf{F}}_{i}^{i n v}=\bar{u}_{i} \overline{\mathbf{Q}}+\left[0, \delta_{1 i} \bar{p}, \delta_{2 i} \bar{p}, \delta_{3 i} \bar{p}, \bar{p} \tilde{u}_{i}\right]^{T}$ and $\overline{\mathbf{F}}_{i}^{v i s}=\left[0, \tilde{\tau}_{1 i}, \tilde{\tau}_{2 i}, \tilde{\tau}_{3 i}, \tilde{\tau}_{k i} \tilde{u}_{k}+\tilde{q}_{i}\right]^{T}$, with the stress tensor $\tilde{\tau}_{i j}$, total energy $\tilde{E}$ and heat flux $\tilde{q}_{j}$ being formulated as

$$
\tilde{\tau}_{i j}=2\left(\mu+\mu_{T}\right)\left(\tilde{S}_{i j}-\frac{1}{3} \frac{\partial \tilde{u}_{j}}{\partial x_{j}} \delta_{i j}\right), \quad \tilde{E}=\bar{\rho} \tilde{e}+\frac{1}{2} \bar{\rho} \tilde{u}_{i} \tilde{u}_{i}, \quad \tilde{q}_{i}=-\left(k+k_{T}\right) \frac{\partial \tilde{T}}{\partial x_{i}}
$$

where the state equation $\bar{p}=\bar{\rho} R \tilde{T}$ defines the relation between pressure, density and temperature for ideal gas. 
For the LES part, subgrid scale (SGS) stress modeling remains under heavy debate in the LES community with, for example, constant coefficient or dynamic coefficient SGS, non-linear models ${ }^{11}$ or using no model, but in conjunction with the numerical dissipation (and other traits) of the scheme (often loosely referred to as Implicit LES) taking place. The attractions of ILES have been discussed widely. Grinstein and Fureby ${ }^{12}$ showed that ILES can capture well some complex jet noise vortex dynamics, because there is no subgrid-scale turbulent viscosity in the two-dimensional shear layer. In order to retain this property, but also have an LES modeling of the three-dimensional structures, the model chosen for the present work is the $\sigma$-model, ${ }^{13}$ in which the subgrid-scale viscosity is defined as

$$
\nu_{S G S}=\left(C_{m} \Delta\right)^{2} \mathcal{D}_{m}(\mathbf{u})
$$

with

$$
\mathcal{D}_{m}=\frac{\sigma_{3}\left(\sigma_{1}-\sigma_{2}\right)\left(\sigma_{2}-\sigma_{3}\right)}{\sigma_{1}^{2}}
$$

where $\sigma_{1} \geq \sigma_{2} \geq \sigma_{3} \geq 0$ are the three singular values of the velocity gradient tensor.

Unlike more standard models, like Smagorinsky (where $\mathcal{D}_{m}=\sqrt{2 S_{i j} S_{i j}}$ ), the $\sigma$-model has, by construction, the property to automatically vanish as soon as the resolved field is either two-dimensional or two-component, including the pure shear and solid rotation cases. In addition, the model generates no subgrid-scale viscosity when the resolved scales are in pure axisymmetric or isotropic contraction/expansion. Finally, it has the appropriate cubic behavior in the vicinity of solid boundaries without requiring any ad-hoc treatment.

\section{II.B. Spatial and temporal discretization}

The flow solver, FLUXp, is based on a cell centered finite volume discretisation for arbitrarily unstructured meshes. More details of the solver can be found in Xia. ${ }^{14}$ Despite the fact that high-order schemes are preferred to minimize dispersive and dissipative numerical errors, several studies suggest that with sufficient mesh resolution DNS and LES can be carried out with second-order schemes. Moreover, due to their efficiency and flexibility in handling complex geometry, second-order schemes have good applicability for industrial applications. Indeed, most high order schemes can give poor performance on highly stretched grids. Hence, second-order spatial schemes with dissipation reduction techniques are employed in this study.

To compute the inviscid flux, Roe's flux difference splitting approximate Riemann solver ${ }^{15}$ is employed at the interface between two neighboring control volumes:

$$
\mathbf{F}=\frac{1}{2}\left(\mathbf{F}_{L}+\mathbf{F}_{R}\right)-\varepsilon \frac{1}{2}|\mathbf{A}|\left(\mathbf{Q}_{R}-\mathbf{Q}_{L}\right)
$$

In the above $|\mathbf{A}|=\mathbf{M}|\mathbf{\Lambda}| \mathbf{M}^{-1}$ is the diagonalizing transform and $\mathbf{A}=\partial \mathbf{F} / \partial \mathbf{Q}$ the Jacobian. Here, following Bui, ${ }^{16} 0.1 \leq \varepsilon \leq 1$ is adopted as an additional parameter to control the amount of upwinding (see Xia et al. ${ }^{1}$ ). A similar technique has also been applied by Shur et al., ${ }^{4}$ where it was cast in a form of blending a purely centered and a fully upwinded flux.

The dual-time integral is employed with the outer physical time discretized by a three-level backward Euler scheme. This leads to second-order temporal accuracy. The inner pseudo time is advanced by a threestage Runge-Kutta scheme. As the outer time is discretized implicitly, larger physical time steps are allowed thus increasing the efficiency compared with explicit time marching.

\section{II.C. Acoustic post-processor}

A common approach applied to jet noise prediction ${ }^{7}$ is to simulate the turbulent jet near field and compute the Ffowcs-Williams and Hawkings (FW-H) integration ${ }^{5,17}$ for the far-field sound.

The surface integral, based on the Ffowcs Williams-Hawkings ${ }^{18}$ equation, is computed. This yields the far-field acoustic pressure fluctuation $p^{\prime}(\mathbf{x}, t)$. Since the noise source is inside the surface (if the surface is large enough and far enough from the jet exit region), a simplification can be made by omitting the volume quadrupole integral. This, as suggested by Shur et al. ${ }^{4}$ and Di Francescantonio, ${ }^{17}$ saves substantial data storage. The integral equation is as follows:

$$
4 \pi p^{\prime}=\frac{\partial}{\partial t} \int_{S}\left[\frac{\rho u_{n}}{r}\right] d S+\frac{1}{a_{\infty}} \frac{\partial}{\partial t} \int_{S}\left[\frac{p_{n r}^{\prime}+\rho u_{n} u_{r}}{r}\right] d S+\int_{S}\left[\frac{p_{n r}^{\prime}+\rho u_{n} u_{r}}{r^{2}}\right] d S
$$



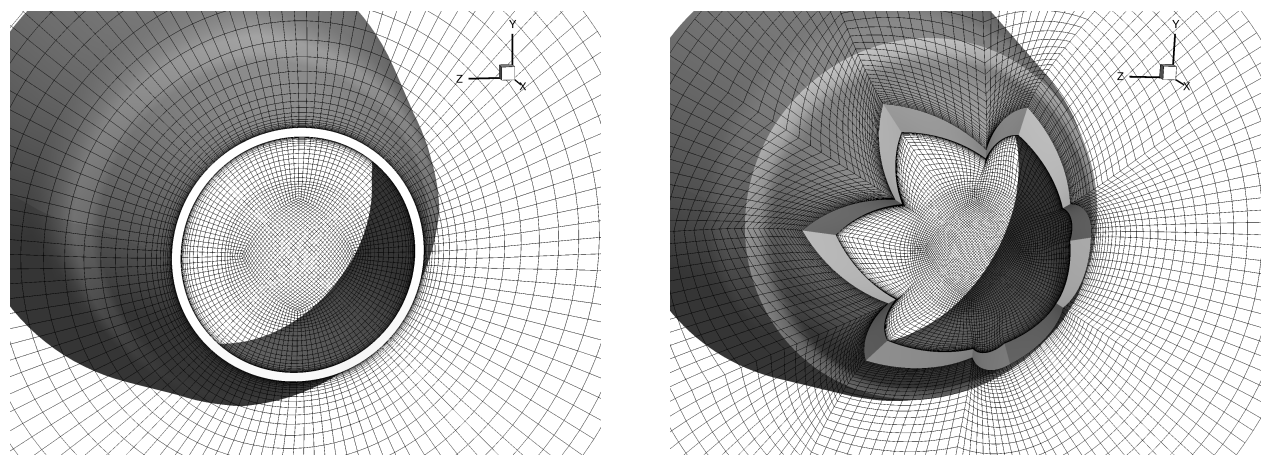

Figure 1. Detail of the nozzle exit for the SMC000 mesh on the left, and the SMC006 mesh on the right.

In the above, $\mathbf{r}$ ( $r$ being its modulus) defines the observer position, $a_{\infty}$ stands for the ambient speed of sound and $S$ is the FW-H surface. The quantities in the square brackets are computed at "retarded" times. Also, $n_{j}$ is the component of the unit outward normal vector on the surface, and $u_{j}$ is the velocity component. Surface data is stored while the simulation is performed, ready for later post-processing. This gives flexibility, avoiding re-running the whole simulation if anything needs to be changed.

\section{Case setup and flow conditions}

The cold jet flow conditions (Test Point 7 of Tanna ${ }^{19}$ ) are specified. These conditions are widely used in jet dynamics and noise experiments with an acoustic Mach number at the jet exit $M a_{a c}=U_{j} / a_{\infty}=0.9$ and a temperature ratio $T_{j} / T_{\infty}=0.84$. The ambient conditions are $p_{\infty}=0.97 \times 10^{5} \mathrm{~Pa}$ and $T_{\infty}=280.2$ K. Reynolds number is around $10^{6}$ based on the nozzle exit diameter $D$ and jet exit velocity $U_{j}$. The axisymmetric nozzle SMC000 has a 2-inch exit diameter while the serrated SMC006, although deviated from SMC000, has a slightly reduced effective jet diameter due to the inward bending of the chevrons. SMC006 is serrated equally in the circumferential direction with six chevron tips and six notches with each chevron corresponding to a $\pi / 3$ sector, and is placed in a position so that planes $z=0$ and $y=0$ cut right through a pair of tips and notches, respectively.

Solutions are obtained on grids ranging from 5 to 10,20, 40 and 80 million grid points. Mesh refinement follows an equal-ratio rule, i.e. the number of cells increases by $\sqrt[3]{2}$ times along all three directions. Hence, for example the $80 \mathrm{M}$ grid is exactly twice as fine as the $10 \mathrm{M}$ grid.

The computational domain consists of the upstream, jet inlet, cylindrical, and downstream boundaries. The domain is $72 D_{j}$ long and expanded to a radial extent of $50 D_{j}$ at the right end. On the nozzle solid wall, the no slip, impermeability velocity and adiabatic thermal conditions are applied. The LES domain comprises non-reflective BCs in the far-field and "sponge" zones with ramped numerical dissipation towards the downstream boundary.

The physical time step was optimized for the $20 \mathrm{M} \operatorname{case}^{20}$ and set to one thousandth flow-through time, $10^{-3} D_{j} / U_{j}$, which technically gives a guaranteed $S t$ number of 1000 from a temporal resolution point of view. The time step refinement follows the same refinement ratio as the cell size, so that it decreases by $\sqrt[3]{2}$ times from a grid to the next. To reach a well developed jet 100-200 flow-through times $t^{*}$ are normally needed and another 50-300t* are further advanced to obtain turbulent statistics and FW-H integral. The choice of the number of $t^{*}$ is dictated by a compromise between accuracy and computational cost. 100 flow-through times are usually considered enough to obtain steady statistics. In the coarser simulations (5 and $10 \mathrm{M}$ cells) $300 t^{*}$ were used, yielding reliable noise results also in the low-frequency range, as will be discussed in Section IV.B. The $80 \mathrm{M}$ case statistics were collected in a period of $50 t^{*}$ in order to support the concept of combination of multiple spectra from affordable simulations. Details of the running cases are summarized in Table 1. 
Table 1. Numerical simulation case summary.

\begin{tabular}{ccccc}
\hline Case ID & Nozzle & Grid points & Time step $\left(\times 10^{-7} \mathrm{~s}\right)$ & Integration time $\left(\# t^{*}\right)$ \\
\hline R005M & SMC000 & $5,156,644$ & 2.734 & 300 \\
R010M & SMC000 & $10,586,144$ & 2.17 & 300 \\
R020M & SMC000 & $20,673,984$ & 1.722 & 100 \\
R040M & SMC000 & $41,073,727$ & 1.367 & 100 \\
R080M & SMC000 & $84,325,770$ & 1.085 & 50 \\
\hline S605M & SMC006 & $5,346,912$ & 2.734 & 300 \\
S610M & SMC006 & $10,409,304$ & 2.17 & 300 \\
S620M & SMC006 & $20,643,273$ & 1.722 & 100 \\
S640M & SMC006 & $40,574,296$ & 1.367 & 100 \\
S680M & SMC006 & $80,331,332$ & 1.085 & 50 \\
\hline
\end{tabular}
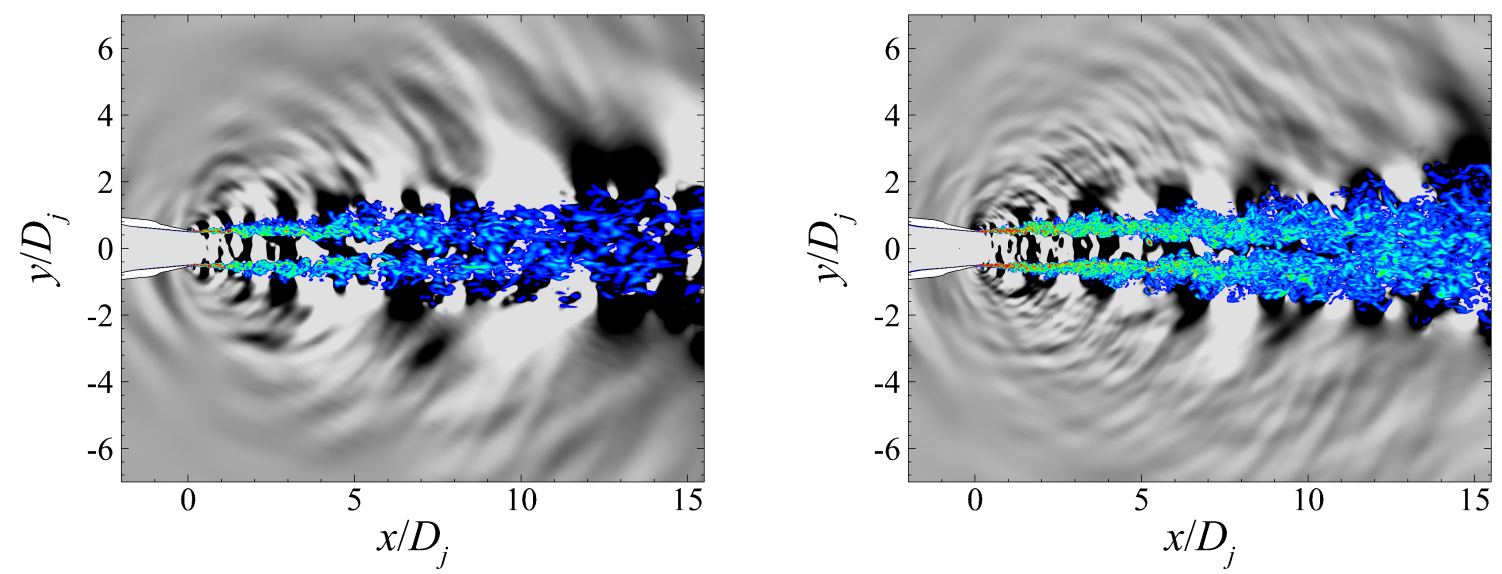

Figure 2. Near-field acoustic wave dilatation visualized by pressure contours (greyscale) and vorticity contours (rainbow). R010M on the left; R080M on the right.

\section{Results and discussion}

\section{IV.A. Instantaneous and mean flow characteristics}

Figure 2 shows pressure and vorticity contours for cases R010M and R080M. Following the refinement rule described above, from $10 \mathrm{M}$ to $80 \mathrm{M}$ the resolution is doubled along every direction. The improvement is evident: the sound waves present higher frequencies (much more detailed shorter wave length structures), while the vorticity contours show smaller structures in both the near-field and the far-field.

A similar comparison for cases $\mathrm{S} 610 \mathrm{M}$ and $\mathrm{S} 680 \mathrm{M}$ is depicted in figure 3, by isosurfaces of the $Q$ criterion, ${ }^{21}$

$$
Q=-\frac{1}{2}\left(\|S\|^{2}-\|\Omega\|^{2}\right)
$$

where $S$ and $\Omega$ denote the strain and rotation tensor of the velocity. The serrated-shape shear produced at the inner lip of the nozzle (visible in red thanks to the transparency of the nozzle geometry) undergoes a faster transition in the $80 \mathrm{M}$ grid, breaking down into noticeably smaller structures. There is however a clear similarity between the blue roller-like big structures of the two cases, which confirms that the coarser grids used in this study are able to capture the large-scale behavior of the jet, despite the lack of smaller-scale information.

Mean quantities are calculated by means of both time and azimuthal averages. Figure 4 compares mean axial velocity and normal Reynolds stress along the centerline and normal stress along the lipline for the round nozzle. Symbols are measurement data from Bridges, ${ }^{22}$ Arakeri, ${ }^{9}$ and Zaman. ${ }^{23}$ The agreement between centerline numerical predictions and experiments is remarkable. The length of the potential core is slightly underpredicted in coarser grids ( $5 \mathrm{M}$ and $10 \mathrm{M}$ ), whereas $40 \mathrm{M}$ and $80 \mathrm{M}$ grids are able to properly 

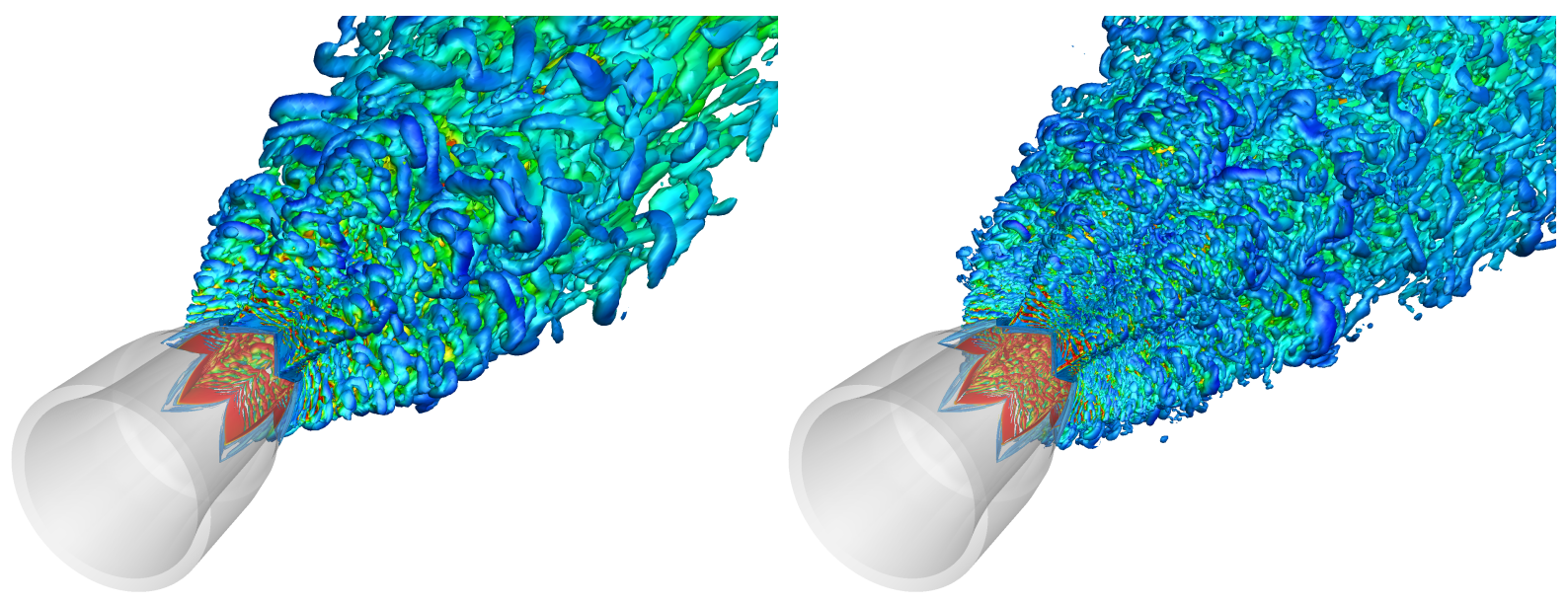

Figure 3. $Q$-criterion isosurfaces of SMC006, colored by $u$. S610M on the left; S680M on the right.
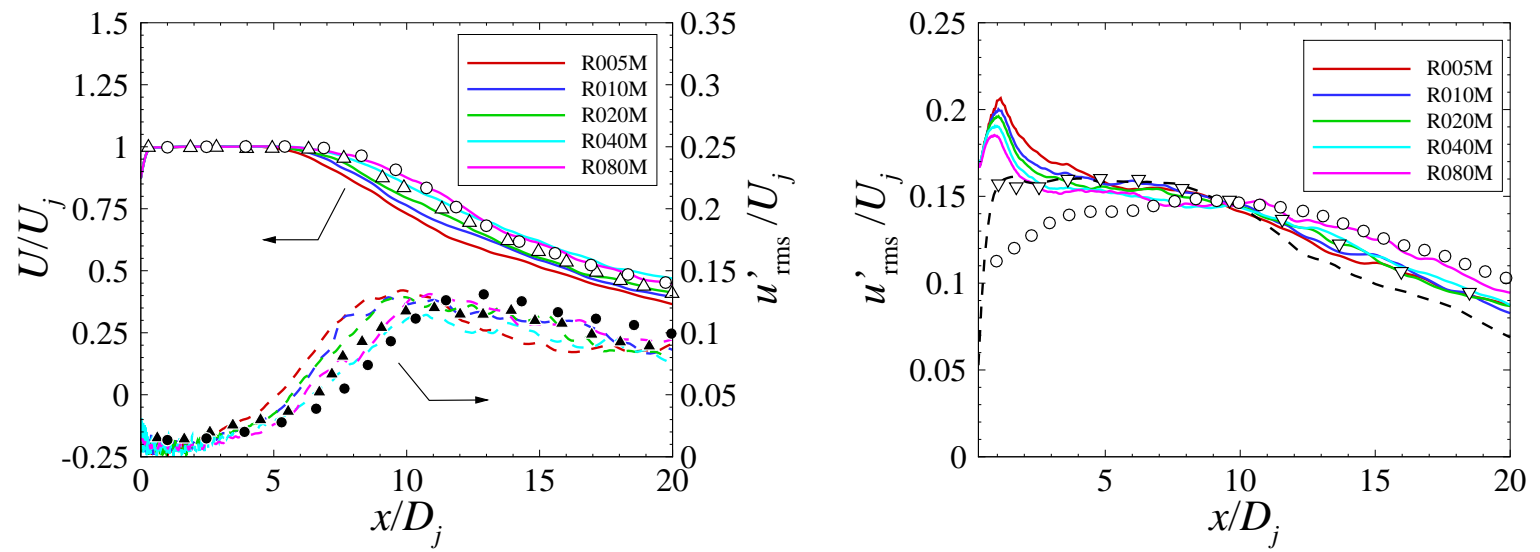

Figure 4. Round Nozzle. On the left: - centerline velocity, - - centerline normal stress, $\mathbf{B r i d g e s}^{24} \boldsymbol{\Delta}$ Arakeri. ${ }^{9}$ On the right: - lipline normal stress, $\bigcirc$ Bridges, ${ }^{24} \nabla$ Zaman, ${ }^{23}--$ preliminary additional study on $\varepsilon$ dependency.

capture even the potential core from Bridges, traditionally considered particularly long. The lipline normal stress shows a typical overprediction compared to Bridges in the proximity of the nozzle lip. We believe this is largely due to the laminar-like (i.e. no resolved turbulent fluctuation) behavior of the boundary layer at the nozzle exit and of the initial numerical shear layer. A better agreement is found with the Ma $=0.5$ case of Zaman, especially when taking into consideration the dependency on the central-upwinding blending parameter $\varepsilon$ (see Section II.B): the result of a preliminary study for $\mathrm{Ma}=0.5$ with a $5 \mathrm{M}$ grid and optimized $\varepsilon$ is depicted in the dashed black line of Figure 4. On the other hand, it is clear that refining the grid has the beneficial effect of reducing the peak normal stress, since it allows for a faster transition to a turbulent shear layer. With the right strategy to introduce boundary layer turbulence inside the nozzle, it should be possible to limit the lipline normal stress even without resorting to extremely fine grids.

For the serrated SMC006 nozzle, the potential core is notably shortened and the simulations are in very good agreement with all grids, as show in Figure 5. This can be interpreted as the result of the enhanced mixing dispersing the momentum of the core jet stream caused by the much increased radial velocity near the exit. The indication is that the flow past tips tends to go inward, whereas the flow through the notches outward (see Figure 3) creating extra streamwise vorticity, hence more mixing, which is captured even by the coarser meshes.

Mean velocity profiles for the serrated case are plotted in Figure 6, for a cut plane through a chevron root (tip cut plane results are omitted here as being less difficult to capture ${ }^{2}$ ). An average is performed on the six periodic azimuthal root planes. Streamwise locations are $x / D_{j}=0.5,1,2.5,5$ and 10 . Individual velocity profiles are separated by a horizontal offset of 1 . They are well captured by all grids, with minor discrepancy 


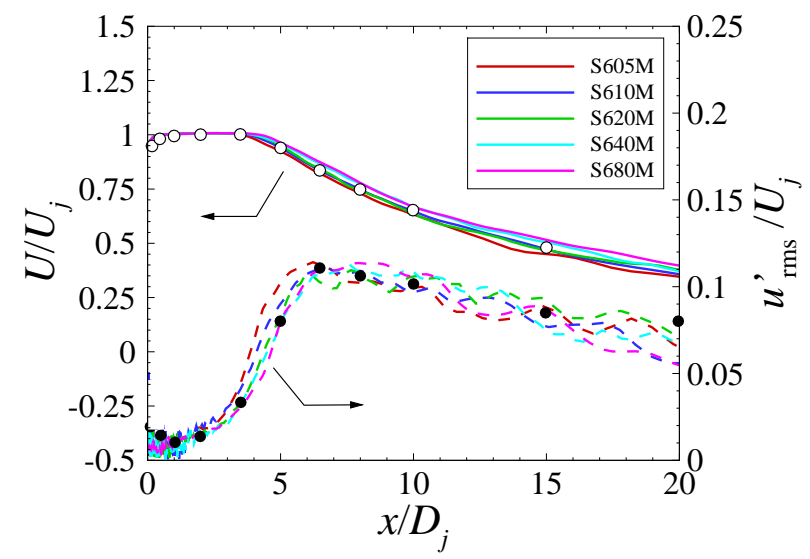

Figure 5. Chevron nozzle. - centerline velocity, -- centerline normal stress, 1 Bridges. ${ }^{24}$
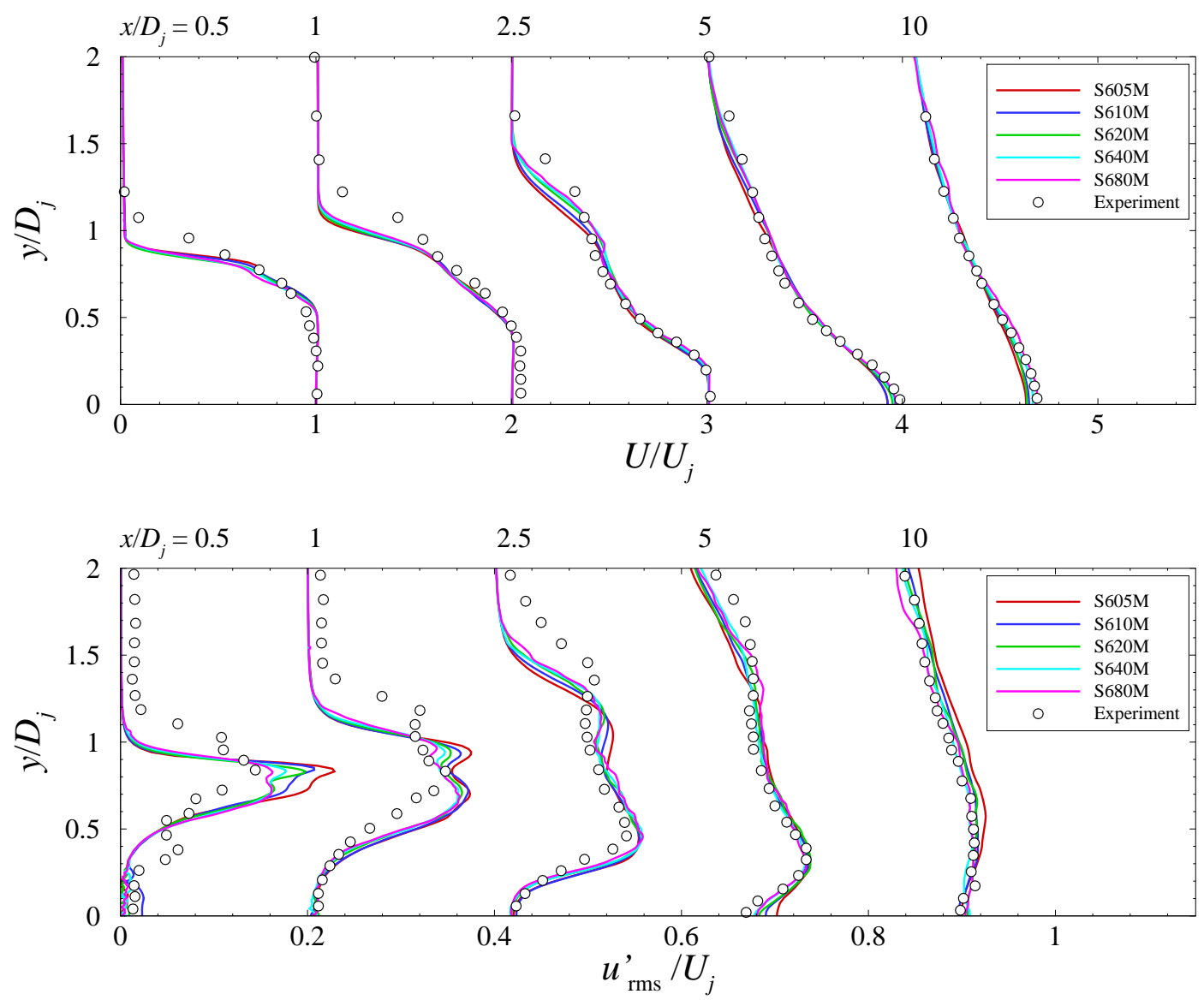

Figure 6. Chevron root cut plane. Top: velocity profiles. Bottom: normal stress profiles. $\bigcirc$ Bridges. $^{24}$ 


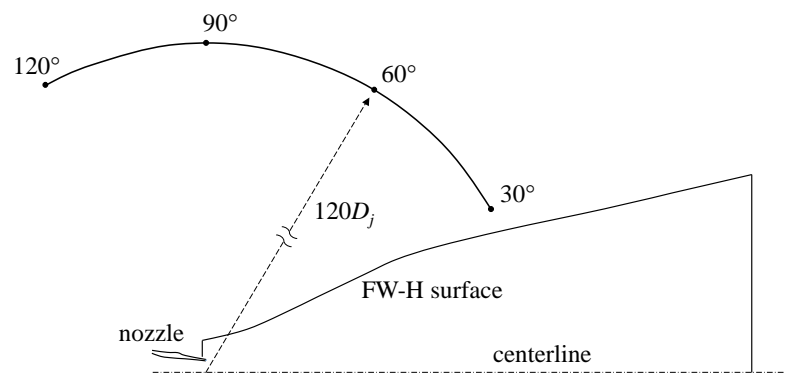

Figure 7. Representation of the FW-H surface profile and the far-field sound observer position.

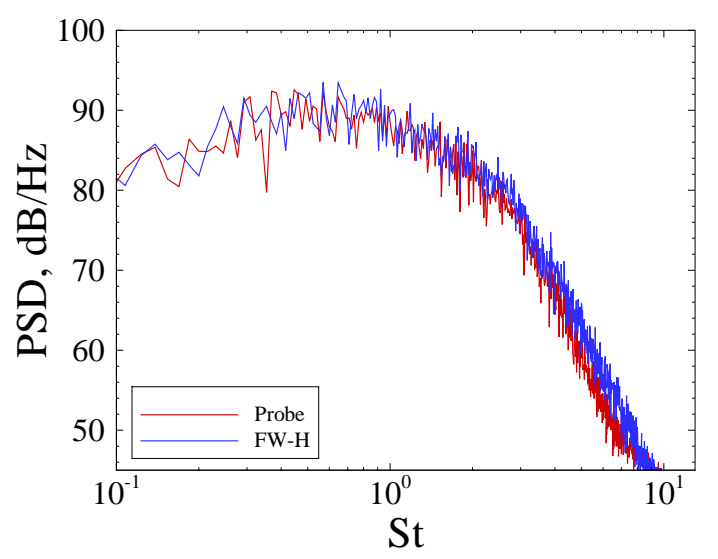

Figure 8. Comparison of the near-field $90^{\circ}$ power spectral density for the chevron nozzle, from an LES probe and from FW-H.

at $x / D_{j}=0.5,1$ and 2.5 in the outer region, where the finer grids behave slightly better. The normal stress profiles are separated by an offset of 0.2 . As the flow through the chevron roots is strongly non-parallel, it is more challenging to predict compared to that along the chevron tips (not shown here). However, all grids are able to capture the two distinct peaks of the normal stress in the first streamwise locations. The grid refinement produces a reduction of the peak values, with the $80 \mathrm{M}$ grid yielding a remarkable agreement with the experiment even at $x / D_{j}=0.5$.

\section{IV.B. Far-field sound}

Figure 7 shows a sketch of the FW-H surface with the sound observer positions. The surface, which is taken from previous studies, ${ }^{1,2}$ is $25 D$ in length, and has diameters of around $3 D$ and $18 D$ at its ends. It is used with upwind and downwind closing discs, the influence of which has not been thoroughly analyzed, as it is beyond the interest of this paper. The FW-H integrals are calculated at $120 \mathrm{D}$ from the nozzle exit center at polar angles $\Theta$.

The FW-H code has been validated by comparing the power spectral density (PSD) received by a nearfield observer close to the FW-H surface, with that detected by a probe directly from the LES simulation. Figure 8 shows the agreement for a $90^{\circ}$ near-field PSD from case S605M.

All the following numerical sound spectra are compared to the experiments of Brown and Bridges. ${ }^{25}$ The simulations with the chevron nozzle yielded a slightly lower effective Mach number of 0.87 , compared to the experimental value of 0.9 ; the noise results were corrected accordingly using Lighthill's 8 th power law.

Figures 9 and 10 show the FW-H power spectral density of the far-field sound at two different polar angles, $\Theta=30^{\circ}$ and $90^{\circ}$, for SMC000 ad SMC006 respectively. As can be seen, at $30^{\circ}$ both nozzles present a slight underprediction at low frequencies and they decay too fast at high frequencies, with the grid refinement not yielding a significant improvement. At $90^{\circ}$ both nozzles present a remarkable agreement up to a cut-off frequency of $\mathrm{St}=2$. Here the improvement due to the grid refinement is clear and consistent, shifting the agreement with the experiment up to $\mathrm{St}=3-4$ for the $80 \mathrm{M}$ case. On the other hand, the $80 \mathrm{M}$ case shows a higher discrepancy in the low-frequency range, due to the shorter integration time $\left(50 t^{*}\right)$. These graphs suggest that the coarser grids used in this paper can capture low-frequency phenomena, with no significant effect caused by the lack of high-frequency resolution. Hence a coarser mesh could be run for a longer time to predict the low-frequency part of the spectrum, while a finer mesh could give more accurate high-frequency results.

In order to assess this statement, a clearer representation can be obtained by integrating the spectra over one-third octave bands. The resulting sound pressure level presents a much more regular spectrum, allowing to determine the influence of the integration time and of the grid refinement.

In Figure 11 one-third octave spectra obtained with different integration times are compared, for the round nozzle with $5 \mathrm{M}$ and $10 \mathrm{M}$ cells. It is clear that only after $300 t^{*}$ the low frequency prediction is reliable, and that all spectra depart from the expected curve for $\mathrm{St}<10 \mathrm{St}_{\min }$, as already shown by Mendez et 

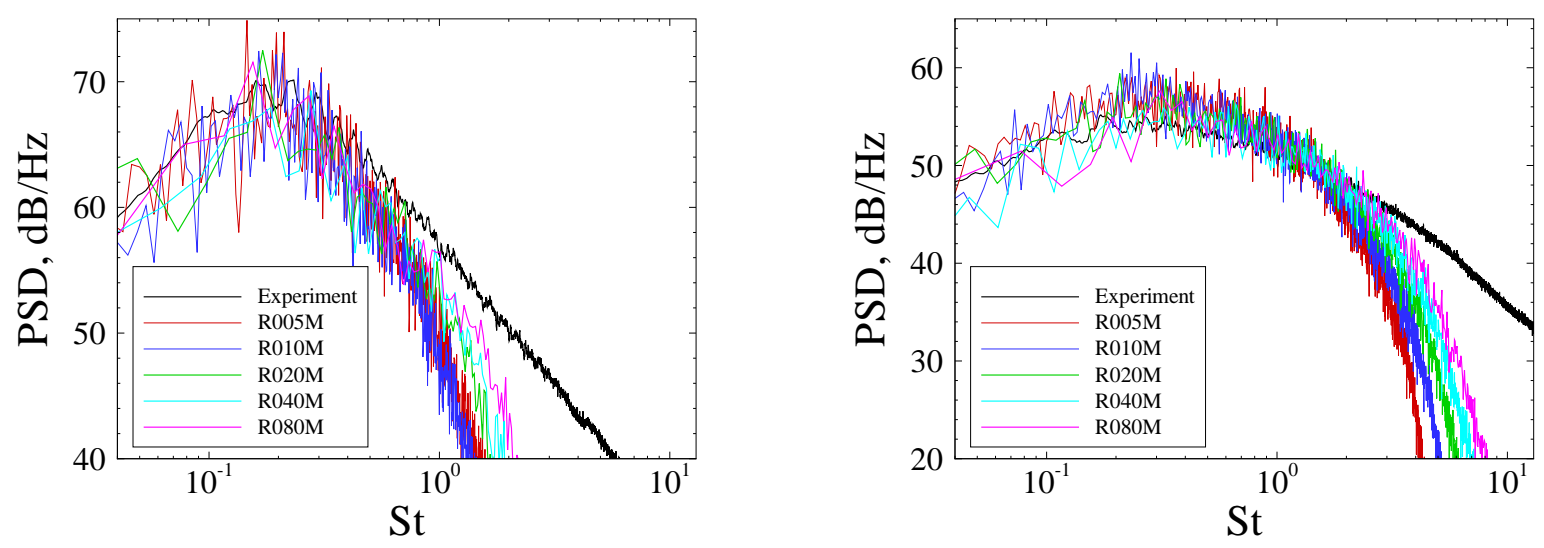

Figure 9. Far-field sound PSD for SMC000 at $R=120 D . \Theta=30^{\circ}$ on the left; $\Theta=90^{\circ}$ on the right.
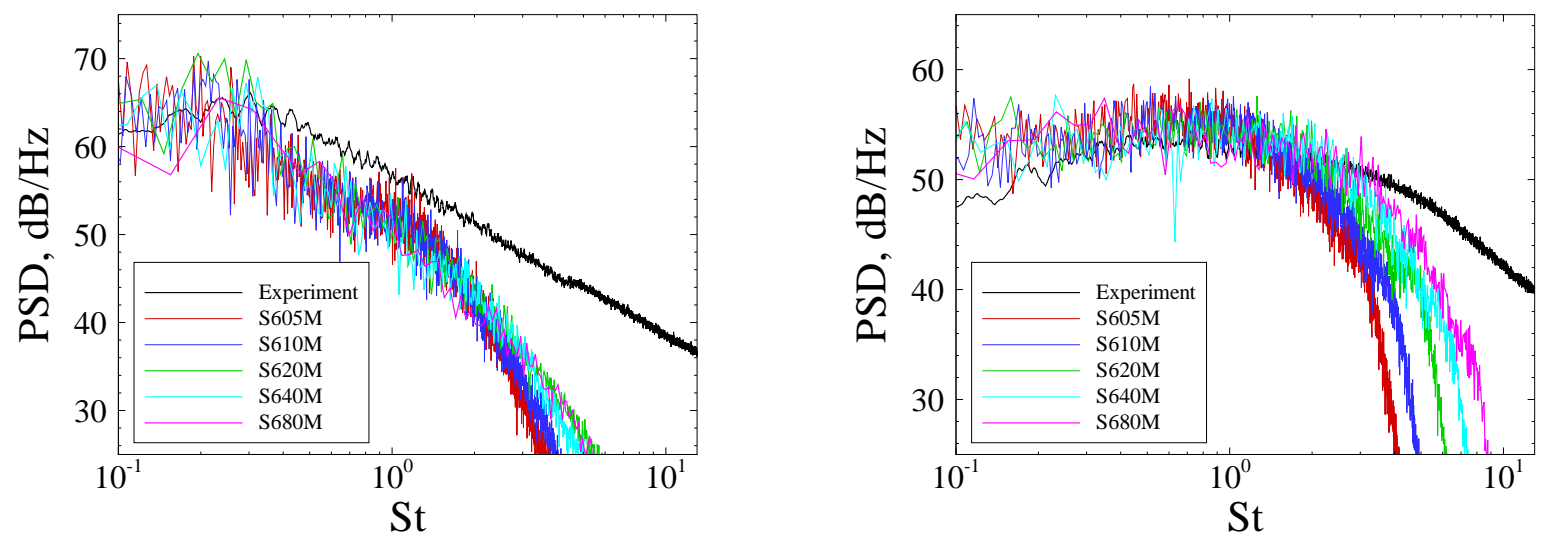

Figure 10. Far-field sound PSD for SMC006 at $R=120 D . \Theta=30^{\circ}$ on the left; $\Theta=90^{\circ}$ on the right.

al. ${ }^{6}$ Hence in the following graphs all spectra obtained with an integration time of $100 t^{*}$ are displayed for St $>0.4$, while the $80 \mathrm{M}$ case $\left(50 t^{*}\right)$ is displayed for $\mathrm{St}>0.8$.

A complete comparison of the one-third octave spectra from all the cases presented in this paper (see Table 1) is depicted in Figure 12. Both the round and the chevron cases show that finer grids are able to capture higher frequencies with a consistent improvement. It is immediately clear that spectra from different grids could be combined to obtain a single broader spectrum. Assuming to run only the $5 \mathrm{M}$ and the $80 \mathrm{M}$ case, the computational cost would be close to that of the latter, being that of the former quite lower even for longer integration times. That would result in a drastic saving, since the finer simulation wouldn't need to capture the low frequencies.

In the frequency range where coarser and finer spectra overlap, a minor difference in SPL can be noticed, with the coarser grids yielding a slightly higher value. This overprediction vanishes towards lower frequencies (see Figure 11). Nonetheless, a combination of different spectra would need to take it into account.

Finally, Figure 13 shows the far-field overall sound pressure level,

$$
\text { OASPL }=20 \log _{10}\left(\frac{p_{r m s}^{\prime}}{2 \times 10^{-5} P a}\right)
$$

The root-mean-square pressure fluctuation $p_{r m s}^{\prime}$ is obtained from the FW-H integrated far-field pressure perturbation $p^{\prime}$ in the time domain. All cases present a remarkable agreement with the experimental results, especially at higher angles. Better predictions for low angles might be gained by tuning the downstream closing disk (partially closing or averaging multiple disks). The grid refinement doesn't seem to produce a consistent improvement, probably due to the previously mentioned overprediction of the coarser cases in 


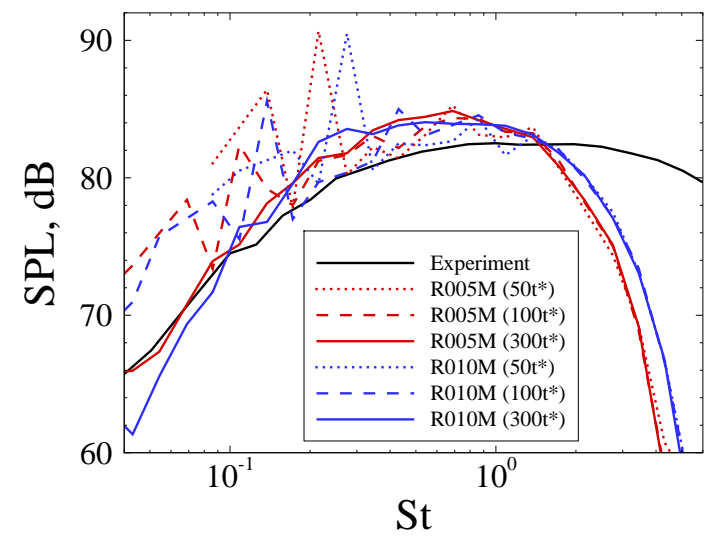

Figure 11. Comparison of $1 / 3$ octave spectra for different integration times.
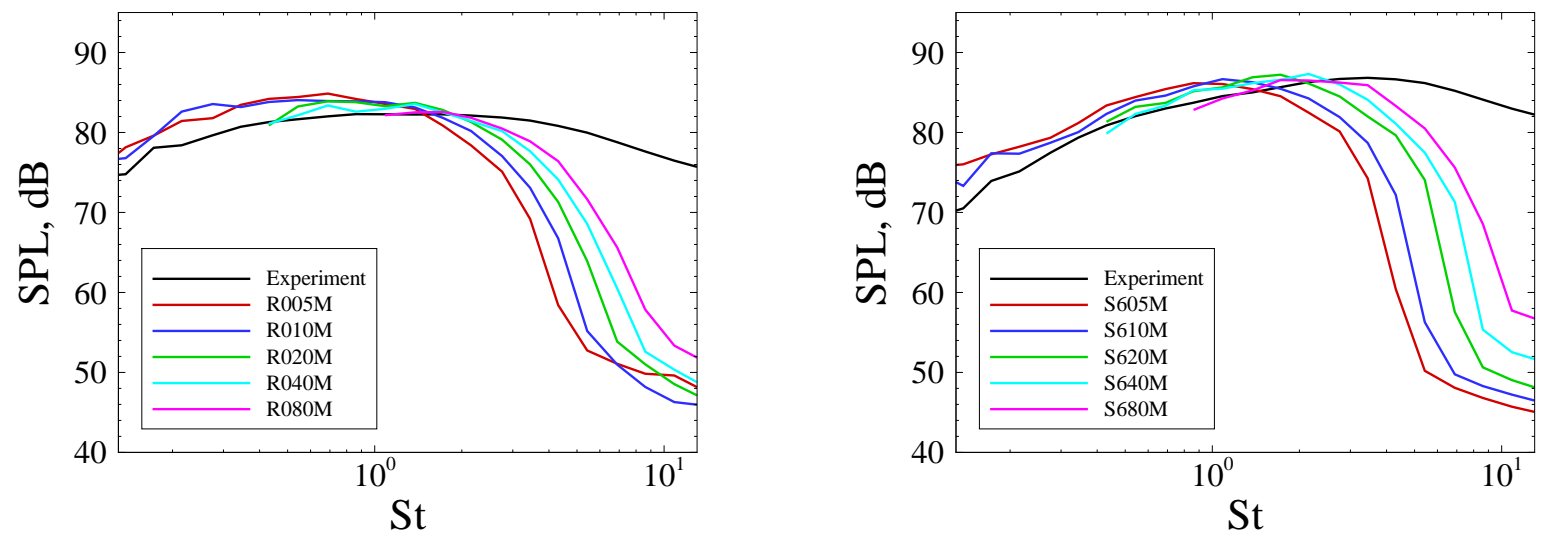

Figure 12. $1 / 3$ octave spectra at $\Theta=90^{\circ}$ for the round nozzle (left) and the chevron nozzle (right).

mid-range frequencies. On the other hand, being able to predict the overall SPL with a coarser grid could in fact be beneficial.

\section{Conclusions}

Large-Eddy Simulations have been carried out for subsonic turbulent jets from an axisymmetric nozzle and a serrated nozzle, with sequentially refined grids. Favorable agreement with the mean and secondorder fluctuating quantities has been broadly gained. The predictions obtained with the finer grids show an impressive agreement with the experimental potential core length, especially considering its traditional underprediction in literature.

Far-field sound spectra obtained with the FW-H technique show that grid refinement yields a clear improvement in the high-frequency range, by raising the cut-off frequency. On the other hand, the longer integration time of the coarser simulations allows to adequately capture the low-frequency range, despite the lack of high-resolution information.

From the analysis of one-third octave results, the possible combination of multiple spectra for widened spectrum prediction has been discussed, with encouraging premises. With this approach, a coarser mesh could potentially be run for a longer time to predict the low-frequency part of the spectrum, while a finer mesh could give more accurate high-frequency results without the need of long and costly simulation times. 

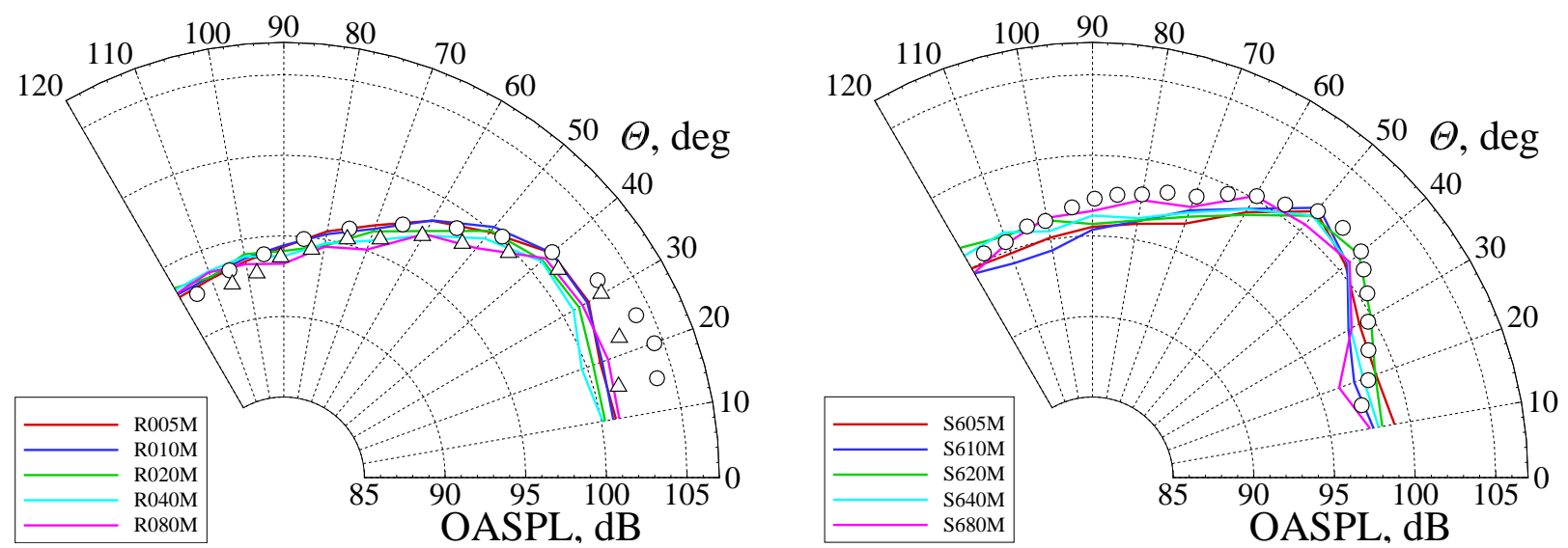

Figure 13. Far-field overall sound pressure level for the round (on the left) and the chevron nozzle (on the right), at $R=120 D$. $\triangle$ measurements of Tanna; ${ }^{19} \bigcirc$ measurements of Brown and Bridges. ${ }^{25}$

\section{Acknowledgements}

This work is financially supported by UK Engineering and Physical Sciences Research Council (EPSRC) under grant number EP/M01391X/1. Computational Allocation Units are provided by the UK national supercomputer, ARCHER, under project e377.

\section{References}

${ }^{1}$ H. Xia, P. G. Tucker, and S. Eastwood, "Large-eddy simulations of chevron jet flows with noise predictions," Int. J. Heat Fluid Flow, vol. 30, pp. 1067-1079, 2009.

${ }^{2}$ H. Xia and P. G. Tucker, "Numerical simulation of single-stream jets from a serrated nozzle," Flow Turbul. Combust., vol. 88, pp. 3-18, 2012.

${ }^{3}$ A. Uzun and M. Y. Hussaini, "Simulation of noise generation in the near-nozzle region of a chevron nozzle jet," AAIA J., vol. 47, pp. 1793-1810, 2009.

${ }^{4}$ M. L. Shur, P. R. Spalart, M. K. Strelets, and T. A. K., "Towards the prediction of noise from jet engines," Int. J. Heat Fluid Flow, vol. 24, pp. 551-561, 2003.

${ }^{5}$ M. L. Shur, P. R. Spalart, and M. K. Strelets, "Noise prediction for increasingly complex jets, Part II: Applications," Int. J. Aeroacous., vol. 4, pp. 247-266, 2005.

${ }^{6}$ S. Mendez, M. Shoeybi, S. Lele, and P. Moin, "On the use of the Ffowcs Williams-Hawkings equation to predict far-field jet noise from large-eddy simulations," International Journal of Aeroacoustics, vol. 12, pp. 1-20, June 2013.

${ }^{7}$ D. Bodony and S. K. Lele, "Current status of jet noise predictions using large-eddy simulation," AIAA J., vol. 46, pp. 364-380., 2008.

${ }^{8}$ J. Bridges and C. Brown, "Parametric testing of chevrons on single flow hot jets," NASA/TM 2004-213107, 2004.

${ }^{9}$ V. H. Arakeri, A. Krothapalli, V. Siddavaram, M. B. Alkislar, and L. M. Lourenco, "On the use of microjets to suppress turbulence in a Mach 0.9 axisymmetric jet," Journal of Fluid Mechanics, vol. 490, pp. 75-98, Sept. 2003.

${ }^{10} \mathrm{C}$. Bogey and O. Marsden, "A study of the grid dependence of the flow field and noise of subsonic jets," American Institute of Aeronautics and Astronautics, Jan. 2016.

${ }^{11}$ Y. Liu, P. G. Tucker, and R. M. Kerr, "Linear and non-linear large-eddy simulations of a plane jet," Comput. Fluids, vol. 37, pp. 439-449., 2008.

${ }^{12}$ F. F. Grinstein and C. Fureby, "Recent progress on MILES for high reynolds number flows," ASME J. Fluids Eng., vol. 124, pp. 848-861, 2002.

${ }^{13}$ F. Nicoud, H. B. Toda, O. Cabrit, S. Bose, and J. Lee, "Using singular values to build a subgrid-scale model for large eddy simulations," Physics of Fluids, vol. 23, no. 8, p. 085106, 2011.

${ }^{14}$ H. Xia, Dynamic Grid Detach-Eddy Simulation for Synthetic Jet Flows. PhD thesis, The University of Sheffield, 2005.

${ }^{15}$ P. L. Roe, "Approximate Riemann solvers, parameter vectors and difference schemes," J. Comp. Phys., vol. 43, pp. 357$372,1981$.

${ }^{16}$ T. Bui, "A parallel, finite-volume algorithm for large-eddy simulation of turbulent flows," NASA/TM 1999-206570, 1999.

${ }^{17}$ P. Di Francescantonio, "New boundary integral formulation for the prediction of sound radiation," J. Sound. Vib., vol. 202, no. 4, pp. 491-509, 1997.

${ }^{18}$ J. E. Ffowcs Williams and D. L. Hawkings, "Sound generated by turbulence and surfaces in arbitrary motion," Philos. Trans. R. Soc. A, vol. A264, pp. 321-342, 1969. 1977.

${ }^{19}$ H. K. Tanna, "An experimental study of jet noise: Part I turbulent mixing noise," J. Sound Vib., vol. 50, pp. 405-428, 
${ }^{20}$ H. Xia, "Turbulent jet characteristics for axisymmetric and serrated nozzles," Computers \& Fluids, vol. 110, pp. 189-197, Mar. 2015.

${ }^{21}$ J. Jeong and F. Hussain, "On the identification of a vortex," Journal of Fluid Mechanics, vol. 285, p. 69, Feb. 1995.

${ }^{22}$ J. Bridges and M. Wernet, "The NASA subsonic jet particle image velocimetry (PIV) dataset," NASA/TM 2011-216807, 2011.

${ }^{23} \mathrm{~K}$. Zaman, "Flow field and near and far sound field of a subsonic jet," Journal of Sound and Vibration, vol. 106, pp. 1-16, Apr. 1986.

${ }^{24}$ J. Bridges and M. Wernet, "Establishing Consensus Turbulence Statistics for Hot Subsonic Jets," American Institute of Aeronautics and Astronautics, June 2010.

${ }^{25}$ C. Brown and J. Bridges, "Small hot jet acoustic rig validation," NASA/TM, vol. 214234, p. 2006, 2006. 